\title{
Alzheimer's Disease: Current and Future Treatments. A Review
}

Evelyn Chou'

\begin{abstract}
Alzheimer's disease (AD) is a currently incurable neurodegenerative disorder whose treatment poses a big challenge. Proposed causes of $A D$ include the cholinergic, amyloid and tau hypotheses. Current therapeutic treatments have been aimed at dealing with the neurotransmitter imbalance. These include cholinesterase inhibitors and N-Methyl-D-aspartate (NMDA) antagonists. However, current therapeutics have been unable to halt AD progression. Much research has gone into the development of disease-modifying drugs to interfere with the course of the disease. Approaches include secretase inhibition and immunotherapy aimed at reducing plaque deposition. However, these have not been successful in curing $A D$ as yet. It is believed that the main reason why therapeutics have failed to work is that treatment begins too late in the course of the disease. The future of $A D$ treatment thus appears to lie with prevention rather than cure. In this article, current therapeutics and, from there, the future of $A D$ treatment are discussed.
\end{abstract}

Keywords: Alzheimer's disease, disease-modifying drugs, beta-secretase inhibitors, gamma-secretase inhibitors, cholinergic, amyloid, tau (Source: MeSH, NLM)

\section{Introduction}

The most common form of dementia is Alzheimer's disease (AD). It is a degenerative and currently incurable terminal disease, affecting about $75 \%$ of the 35 million people worldwide suffering from dementia. It is predicted that the prevalence of $A D$ will double every 20 years, meaning an estimated $115 \mathrm{mi}-$ Ilion individuals may be suffering from $A D$ by 2050.' $A D$ is thus becoming increasingly recognized as a major cause of medical and social burdens in the elderly population worldwide. ${ }^{2}$ In its preclinical stages, AD cannot be diagnosed, while its clinical stages are characterized by impairment of cognitive functions (i.e. recent memory, language difficulties, spatial disorientation and visual agnosia), with behavioural disturbances significant enough to compromise activities of daily living (ADLs). Life expectancy is reduced, with patients generally living up to 5-8 years following diagnosis. ${ }^{3}$

Currently, no drugs are available to halt the progression of neurodegeneration in $A D$; the nature of $A D$ treatment is symptomatic. $^{2}$ For instance, cholinesterase inhibitors (Cls) that promote cholinergic neurotransmission are used in mild to moderate cases of AD. Memantine, an N-methyl-D-aspartate (NMDA) receptor antagonist, is used in moderate to severe cases to prevent excitotoxicity, ${ }^{4}$ and antipsychotics and antidepressants are used in the treatment of neuropsychiatric symptoms. ${ }^{5}$

The future of treatment of $A D$ lies in the targeting of neuritic plaques (NPS) and neurofibrillary tangles (NFTs), which has the potential to delay neurodegeneration. ${ }^{6}$

The daunting statistics and the impacts that AD has on suffe- rers, caregivers and the society make it exceptionally vital that we review how AD is currently being treated and how this is likely to change in the near future with the possible development of disease modifying treatments.

\section{Search Strategy and Selection Criteria}

The papers for this review article were identified by computerized advanced searches in Pubmed database and Google Scholar using the keywords 'Alzheimer's', 'beta-secretase' and 'gamma-secretase'. These papers included meta-analyses, original research articles, review articles and clinical trials. Information was also obtained from textbooks and Alzheimer's disease forums. This review follows the Preferred Reporting Items for Systematic Reviews and Meta-Analyses (PRISMA) Statement.?

\section{Clinical Features}

The progression of $A D$ can be divided into a series of stages: pre-dementia, mild, moderate and severe.

The pre-dementia stage is often unreliably distinguished from normal aging or stress-related issues. ${ }^{3,8}$ One of the first signs is the deterioration of episodic memory. No decline in sensory or motor performance occurs at this stage, and other aspects such as executive, verbal and visuospatial functions are slightly impaired at most. An individual remains independent and is not diagnosed as suffering from AD. ${ }^{8}$

During mild stages of $A D$, increased memory loss affects recent declarative memory more profoundly than other capacities, such as short-term, declarative and implicit memories. ${ }^{3}$ 
Motor function remains normal, and sensory performance is not impaired extensively. However, some visual, auditory and olfactory functions may be affected. ${ }^{8}$ Communication begins to decline as patients find themselves unable to recall certain words. The individual may be able to remain independent, but not without some assistance. ${ }^{3,9}$

Recent memory continues to deteriorate in the moderate stage. Due to an inability to create new memories, AD patients seem to live in the past. 9 Patients are still able to manage basic ADLs, but help is required in certain areas such as grooming and dressing. ${ }^{3.9}$ Insight into their disease is commonly lost by this stage, with patients becoming delusional. A longitudinal study conducted in 1993 showed that it is at this stage that cognitive decline, aggression, depression and incontinence in patients become predictive factors for placement in nursing homes..$^{10}$

In the severe stage, even early memories can be lost. Basic ADLs are now affected, declining gradually. Communication deteriorates further to single words or phrases, and language is thus significantly impaired. ${ }^{3.9}$ Behavioural disturbances occur, causing disruptions to caregivers. ${ }^{3,11}$ The most common cause of death in $A D$ patients is pneumonia, ${ }^{12}$ followed by myocardial infarction (MI) and septicaemia. ${ }^{3}$

\section{Risk Factors}

Inheritance of certain genes is a risk factor for $A D$, with both familial and sporadic cases occurring. In sporadic AD, which is the more common form, there is a link with the apolipoprotein $\varepsilon_{4}$ (APOE4) allele, with the risk being greater in homozygotic situations. ${ }^{1,13}$ It has been shown that transgenic mice expressing either mouse or human apoE develop neuritic plaques (NPS) associated with neuritic degeneration due to fibrillar amyloid- $\mathbb{B}$ deposits. $n$ contrast, in apoE negative mice, no neuritic degeneration was observed despite the presence of non-fibrillary amyloid-B deposits. ApoE thus appears to play a critical role in the progression of NPs and degeneration. ${ }^{14}$

Environmental factors also contribute to the development of sporadic AD. ${ }^{15}$ Familial AD, on the other hand, has been associated with mutations in presenilin 1 (PSEN 1 ) and presenilin 2 (PSEN2), as well as the amyloid precursor protein (APP) gene, which is located on chromosome 21.,13 Many other candidate polymorphic genes have been associated with increased $A D$ risks, including secretase, peptidase, microtubule, cytoskeletal, anti-apoptotic and protease genes.'

Vascular factors seem to affect the risks of developing AD. Metabolic syndrome,' comprising hypertension, dyslipidaemia, obesity and diabetes mellitus, has been associated with increased risk. ${ }^{16}$ Hypertension contributes to the formation of neuritic plaques (NPS), neurofibrillary tangles (NFTs), and characteristic lesions seen in AD. ${ }^{17}$ Dyslipidaemia and diabetes mellitus are not only implicated in the generation of NPs, but also cause cerebrovascular dysfunction. ${ }^{18}$ In addition, obesity has been linked to cognitive decline and resistance to insulin the latter of which leads to hypertension, diabetes and cerebrovascular dysfunction. ${ }^{19}$

Psychosocial factors are also implicated in AD. With greater social, physical and mental stimulation, individuals are less likely to develop AD later in life. The risk of cognitive decline is higher in those with lower physical activity. Additionally, the protective effect of physical activity is more prominent in apoE4 allele carriers. These three lifestyle factors appear to act together in a common pathway in their protection against dementia.,20

\section{Causes}

\section{Cholinergic Hypothesis}

The cholinergic hypothesis of AD came about due to the combined observations of deficits in choline acetyltransferase and acetylcholine (ACh) and the fact that ACh is important in memory and learning. It was thought that reduction in cholinergic neurons as well as cholinergic neurotransmission led to the decline in cognitive and noncognitive functions. Cholinergic function loss correlated to cognitive decline, but no causal relationship was established. ${ }^{2,21}$ Moreover, the use of cholinesterase inhibitors $(\mathrm{Cls})$ does not have a significant effect in more than half of $A D$ patients receiving treatment, indicating the presence of other important processes in the progression of the disease. ${ }^{21}$

\section{Amyloid Hypothesis}

Amyloidosis is the abnormal deposition of amyloid proteins in tissues, with the altered amyloid proteins forming an insoluble ß-pleated sheet. Reduced tissue and cellular clearance is observed in amyloid protein deposits. The membrane protein amyloid-B precursor protein (APP) is proteolysed to form $A B$, and it is the amyloid form of $A ß$ that makes up the amyloid plaques (neuritic plaques) found in the brains of AD sufferers. ${ }^{6}$

According to the amyloid hypothesis, the basis of $A D$ is the presence of Aß production in the brain. ${ }^{2}$ Evidence for the amyloid hypothesis was compelling, as gene mutations encoding the amyloid- $\$$ precursor protein (APP) was found to cause familial $A D$, with sites of major mutations found in $\gamma$ secretase and $A P P{ }^{6} A B$ is derived from APP by proteolysis in the amyloidogenic pathway, mediated by $B$ secretase (BACE 1 ) and $\gamma$ secretase, in the extracellular and transmembrane region, respectively. Cleavage by $B$-secretase produces APPsß and C99. C99 is further cleaved by $\gamma$ secretase to form either $A B_{1-40}$ or the more hydrophobic, aggregation-prone Aß1-42. ${ }^{22}$

Aß40 is more predominant in cerebral vasculature. ${ }^{2}$ APP can also be cleaved by $\alpha$ secretase in the non-amyloidogenic pathway, producing APPS $\alpha$ and C83. Further evidence came from an experiment in the 1990s whereby transgenic mice expressing three different isoforms of mutant APP were found to have characteristic AD neuropathologies. ${ }^{23}$

Despite widespread support of Aß fibrils being the main cause of pathology seen in $A D$, it was suggested that oligomerization of $A ß 1-42$ plays a more important role. Oligomerization of $A B_{1-}$ 42 produces soluble $A ß$ oligomers which are known as Aß-derived diffusible ligands (ADDLs). Experiments showed that these ADDLs are potentially more toxic than Aß fibrils as they target synaptic spines and disrupt synaptic plasticity, thus affecting cognitive function. Their toxicity lies in toxin receptors on cell surfaces and in Fyn, a tyrosine kinase receptor overexpressed in $\mathrm{AD}$ (Figure 1). ${ }^{24,25}$ 
Figure 1. The Amyloid Hypothesis: Amyloidogenic and Non-amyloidogenic Pathways.

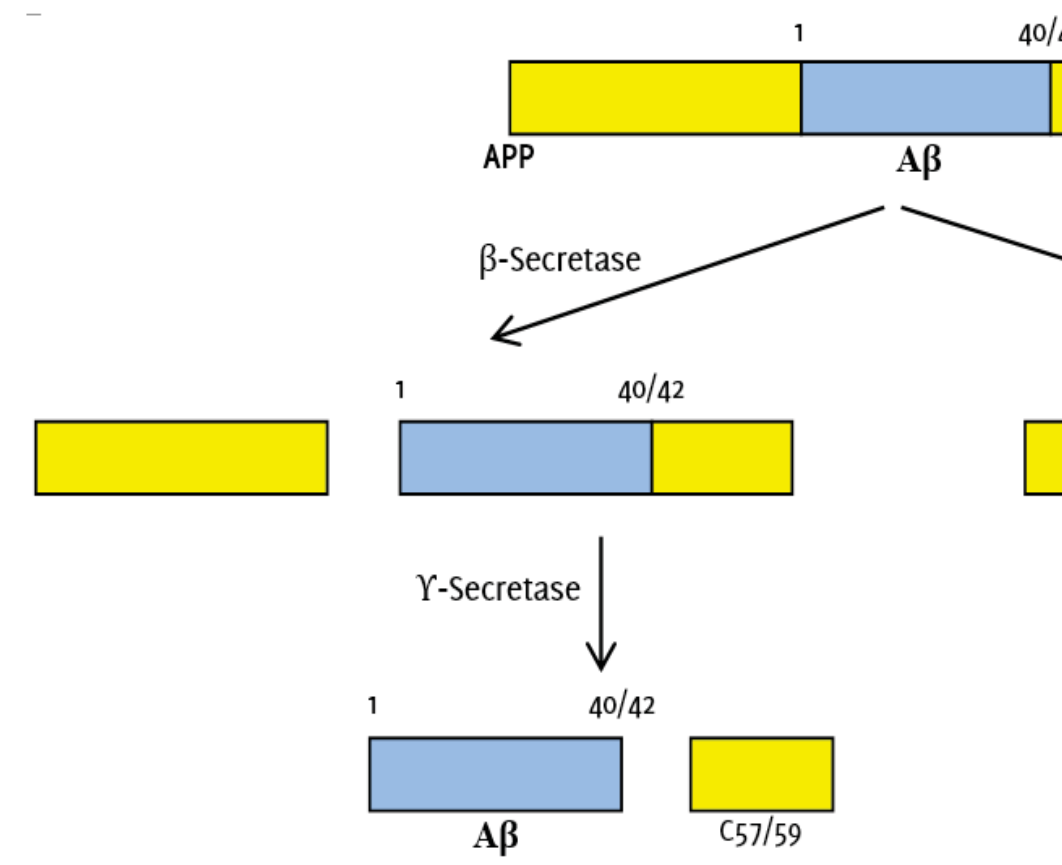

\section{Amyloidogenic pathway}

\section{Tau Hypothesis}

The Tau hypothesis revolves around the presence of neurofibrillary tangles (NFTs) in AD. As a result of increased phosphorylation of Tau (originally bound to microtubules), there is an increase in free tau accompanied by loss of functioning microtubules. ${ }^{26}$ Phosphorylated Tau are subunits of paired helical filaments (PHFs), which form NFTs. The impaired microtubules affect axonal transport of proteins and eventually cause neuronal death. ${ }^{27}$

\section{Neuropathology}

The degeneration of neurons and synapses, declining cholinergic function, characteristic neuropathologic lesions of neuritic plaques (NPS) and neurofibrillary tangles (NFTs) all contribute to cognitive decline. ${ }^{28}$ The neocortex and hippocampus, which are the regions of higher function, are most affected by these lesions. ${ }^{21}$ NPs consist of aggregations of amyloid-ß peptide, while NFTs are located within neurons and their projections and are composed of filamentous hyperphosphorylated tau. The distinction between NPs and NFTs being causative or mere markers of $A D$ and the chronological order in which the pathologies appear are important to the understanding of AD pathology. ${ }^{6}$ A study carried out to determine the role of NPs and NFTS in AD established that NP deposition occurs in early stages of the disease, but does not correlate with progression of illness once clinical stages have been established, whereas NFTs seem to correlate to decline in cognitive function in later stages. ${ }^{28}$

\section{Current Symptomatic Treatments}

It is through the understanding of the disease processes that underlie $A D$ that targets can be ascertained and treatments developed.

\section{Non -amyloidogenic pathway}
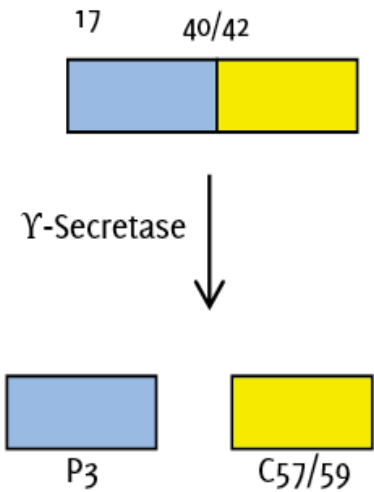

\section{Cholinesterase Inhibitors}

Cholinesterase inhibitors $(\mathrm{Cl})$ aim to increase acetylcholine availability in synaptic neurotransmission in order to treat memory disturbances. Currently, three $\mathrm{Cls}$ are being used as the first-line treatment in mild to moderate $\mathrm{AD}$ : donepezil, rivastigmine and galantamine. ${ }^{2}$ While donepezil and rivastigmine are both selective inhibitors, galantamine inhibits both $\mathrm{ACh}$ and butyrylcholinesterase. A meta-analysis collaborating 13 randomized, double-blind trials that were designed to evaluate the effectiveness and safety of $\mathrm{Cls}$ showed no improvement in ADL and behaviour. In addition, donepezil and rivastigmine showed no significant difference in their impact on cognitive functions, ADLs and behaviour. Overall, similar benefits were observed across all three drugs. ${ }^{29}$ It is known that Cls are unable to halt disease progression, but they have been found to have effects for a substantial period of time. As seen in a randomized double-blind trial, patients undergoing long-term treatment with donepezil showed no beneficial loss for up to two years. ${ }^{30}$

In addition, there may be some added benefits to increased doses of $\mathrm{Cls}$ given. In a randomized, double-blind, parallel-group, 48-week study conducted to determine the efficacy and safety of a rivastigmine patch of a higher dose, deterioration of ADLS was significantly reduced and Alzheimer's Disease Assessment Scale-cognitive subscale (ADAS-cog) was improved in patients treated with higher doses. ${ }^{31}$ Side effects as a result of Cls are minimal and are usually limited to gastrointestinal symptoms such as diarrhea, nausea and vomiting. ${ }^{13}$

\section{NMDA Receptor Antagonists}

Memantine is a non-competitive NMDA receptor antagonist effective in the treatment of moderate-to-severe AD. The modu- 
lation of NMDA receptors results in reduced glutamate-induced excitotoxicity. Its benefits were proven in a 28 -week, double blind, parallel-group study which showed that treatment significantly reduced deterioration in patients. Most adverse reactions to the drug were not severe and were considered to be unrelated to the drug. The positive effect on cognitive function translates to behavioural improvements: patients were less agitated and required less assistance from caregivers. ${ }^{32}$ Improvement of the behavioural and psychological symptoms related to dementia (BPSD) was also highlighted by a meta-analysis of 6 studies involving memantine treatment. ${ }^{33}$

\section{Antidepressants and Antipsychotics}

BPSD is a common occurrence in AD and a major source of burden on caregivers. Cls and memantine help to control these symptoms to a certain extent, but as patients continue to deteriorate, control by these drugs becomes insufficient. ${ }^{2}$

Depression is very common, especially in the early and late courses of the disease. Antidepressants such as: selective serotonin reuptake inhibitors (SSRI: citalopram, fluoxetine, paroxetine, sertraline, trazodone), tricyclic agents and combined serotonergic and noradrenergic inhibitors may be used to counter this. ${ }^{2,34}$ Discontinuation of antidepressants in demented patients in a double blinded, randomized, parallel-group placebo controlled trial showed significant increases in depression when compared to those who continued treatment. These results are indicative of the beneficial effects of antidepressants. ${ }^{34}$

Atypical antipsychotics used in $A D$ include olanzapine, quetiapine and risperidone, which are used to treat psychosis and agitation. However, the use of such drugs appears to be controversial, with patients showing significant declines in cognitive function with antipsychotic drugs administration when compared to patients receiving the placebo. ${ }^{35}$

\section{Disease-Modifying Treatments}

While symptomatic treatments have proven helpful, it is the finding of a cure that is most vital. Since the amyloid hypothesis indicates that $A ß$ generation and deposition from overexpressed APP cleavage make up the fundamental basis of $A D$, interest centers on anti-amyloid therapies. These therapies result in decreased production of $A B$, increased clearance of $A B$ and the prevention of $A B$ aggregation into amyloid plaques. ${ }^{6,36}$ Immunotherapy has also been an area of interest as it targets the clearing of $A B$ peptides, which can either directly or indirectly impact cognitive decline. ${ }^{37}$

Focusing on decreasing Aß generation, several methods can be employed to achieve this, mainly by targeting the amyloidogenic and nonamyloidogenic pathways. B and $\alpha$ secretases both compete for APP, with $B$ - and $\gamma$-secretase processing ultimately resulting in amyloid deposition and $\alpha$-secretase generating soluble APPsa. ${ }^{2}$ Inhibiting $B$ - and $\gamma$-secretases while simultaneously potentiating $\alpha$-secretase action would thus reduce $A B$ generation and deposition overall.

\section{Decreasing $A ß$ Generation}

$\beta$-Secretase Inhibitors

The cloning of $ß$-secretase has allowed for the investigation of its structural and catalytic properties. ${ }^{38}$ Development of clinicaIly effective inhibitors depends greatly on this detailed knowle-

Figure 2. Possible Therapeutic Targets in Anti-amyloid Therapies

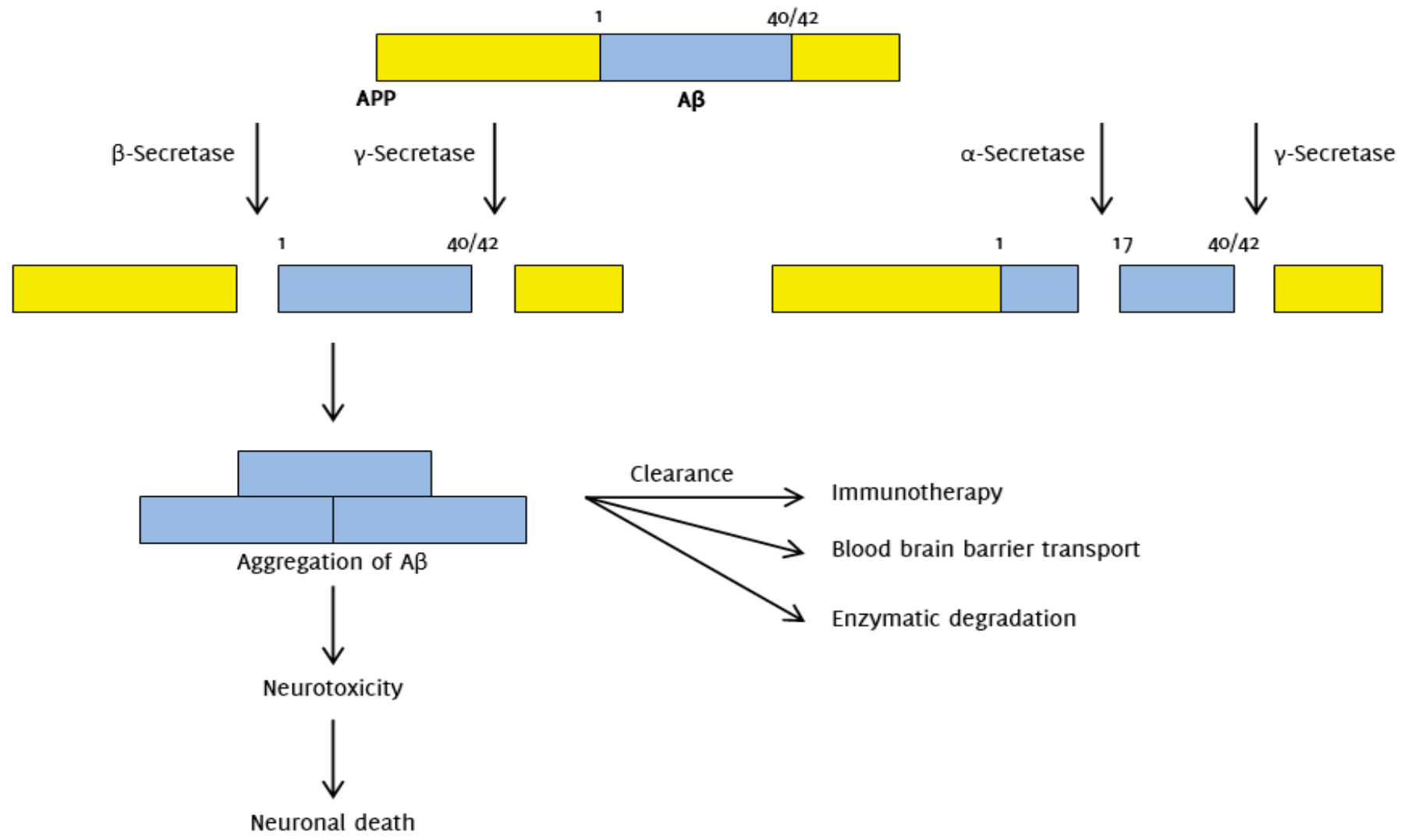


dge of its structure. ß-secretase is a rate-limiting transmembrane aspartyl protease beta-site APP-cleaving enzyme (BACE1) involved in the first proteolytic step of the amyloidogenic pathway of Aß generation. BACE1-deficient mice were used to determine the role of $B$-secretase. In mice with BACE1-/- neurons, generation of $A B_{40}$ and 42 was eradicated, thus confirming the role of BACE1 in the amyloidogenic pathway. ${ }^{39}$ From this, it can be inferred that inhibition of BACE1 activity would be able to reduce $A B$ levels and ultimately reduce neuritic plaques. Thus, it has become a primary therapeutic target.

The close resemblance of B-secretase's catalytic apparatus to other aspartyl protease targets such as HIV protease has contributed to the development of its inhibitors. ${ }^{39}$ However, the secretase's hydrophilic, long and shallow active site presents some problems to the development of its inhibitors. An effective inhibitor would need to be able to penetrate the blood-brain-barrier (BBB) and the cell membrane of neurons. This has made the discovery of small yet potent inhibitors more difficult. ${ }^{36}$ Another challenge is that $B$-secretase inhibitors act as substrates for P-glycoprotein that transports them out of the brain. Efforts have been made to overcome this through the design of inhibitors with higher selectivity for BACE1 over memapsin 1 (BACE2) and cathepsin D (cathD). 2,39 The low number of drugs reaching clinical trials reflects the difficulty of these challenges faced (Figure 2).

The earliest BACE1 inhibitors were peptidic, large, polar and clinically ineffective. These have been progressively improved and they have been made less peptidic. The first orally bioavailable drug-like non-peptidic BACE1-inhibitor LY28113776 was found to significantly lower $A B$ protein in animal models. Healthy volunteers later treated with the compound also demonstrated this decrease in AB levels. Its safety, tolerability and efficacy were determined in a double blind, placebo controlled study in which subjects were assigned either the active drug or a placebo and participated in CSF and plasma sampling. The drug was discontinued due to its pathological effect on accumulations of autofluoroscent material within the retinal epithelium, causing them to be enlarged. Although the drug did not go on to enter the later clinical trial stages, data recorded nevertheless provides support for BACE1 as a target for BACE1 inhibitors. ${ }^{40}$

Despite support for BACE1 inhibitors, certain concerns were yet to be dealt with. In particular, it was not known whether BACE1 played other important physiological roles apart from being involved in the amyloidogenic pathway. ${ }^{40}$ In a 2006 study, it was discovered that BACE1 is involved in the myelination process of peripheral and central nerves. In BACE1-null mice, hypomyelination occurred. This hypomyelination was thought to be due to the role of BACE 1 in the cleavage of neureguin-1 type III, a myelination initiator. At this point, it was still unclear whether neuroglenin-1 cleavage is required for myelin sheath maturation, which would make BACE1 inhibitors potentially dangerous. ${ }^{41}$ However, it was later found that neuroglenin1 signalling is in part independent of BACE1 function. Even though BACE1's processing of the gene does produce a myelin-inducing signal, this signal is not essential for myelination stimulation..$^{42}$

The first ß-secretase inhibitor to enter Phase I clinical trials was announced in 2008. ${ }^{2}$ This compound, CTS-21166, developed by CoMentis, was able to significantly lower Aß levels. It was not only potent (1.2-3.6 nm), but also selective. In cellular assays performed, CTS-21166 did not bind to 60 other enzymes. ${ }^{38,40}$ This was an achievement, as non-specific binding was one of the major issues in developing a clinically effective $B$-secretase inhibitor. It also displayed desirable brain penetration, another problem associated with the development of other inhibitors (Strobel G. Keystone Drug News: CoMentis BACE inhibitor debut. http://www.alzforum.org/new/detail.asp?id=1790. Cited 2013 Sep 30). The injection of CTS-21166 into transgenic mice resulted in reduction of AB 40 and 42 by an average of $36.5 \%$ as well as reduction in amyloid plaques in hippocampal and cortical areas by about $40 \%$. Using this knowledge, the compound was then tested on healthy male volunteers for its tolerability and safety in 6 volunteers, with a range of doses $(7.5-225 \mathrm{mg})$ given intravenously. Results were positive, showing good tolerability over the range of doses administered and slow drug clearance (Strobel G. Keystone Drug News: CoMentis BACE inhibitor debut). Significant plasma $A B$ inhibition continued for up to 72 hours, with recovery to normal levels by 144 hours after the inhibitor was administered. ${ }^{39}$ Later phase clinical trials have yet to be published.

\section{$\gamma$-Secretase Inhibition}

$\gamma$-secretase inhibition is perhaps the most widely and frequently studied mechanism to reduce $A ß$. It is involved in the second step of the amyloidogenic pathway and is thus crucial in $A B$ generation. Another product derived from $\gamma$-secretase cleavage is the amyloid intracellular domain (AICD), which may have a role in gene expression downstream. ${ }^{6} \gamma$-secretase is an intra-membrane cleaving protease, consisting of 4 components: presinilin (PS), nicastrin (NCT), presenilin enhancer (Pen2) and anterior pharynx defective (Aph1). These 4 components were thought to be essential for the activity of $\gamma$-secretase, and the loss of any of the proteins appeared to abolish the activity of $\gamma$-secretase. However, in NCT knock-out mice, it was observed that a complex of PS1, Pen2 and Aph1a was able to function as a $\gamma$-secretase inhibitor, which indicated that NCT is not required for substrate recognition in $\gamma$-secretase. Instead, it is thought to have a role in the stabilization of the enzyme. This makes it much more complex than $B$-secretases. ${ }^{6,43}$ Even though NCT may not be the most important component, $\gamma$-secretase activity is PS dependent. It was the combined knowledge of mutations in PS1 and PS2 resulting in early-onset of $A D$ and PS mutations also causing increased levels of $A B$ that led to the belief that PS has a direct impact in $\gamma$-secretase mediated cleavage of APP. ${ }^{44}$ In an investigation of PS-1 deficient mouse embryos, the cleavage of APP by $\gamma$-secretase was prevented, causing $A ß$ levels to be significantly reduced, whereas cleavage by $\beta$ - and $\alpha$-secretase was unaffected. ${ }^{45}$ This thus confirmed the function of presenilin in $\gamma$-secretase.

Despite $\gamma$-secretases' complexity, the development of $\gamma$-secretase inhibitors has been easier than the development of ß-secretase inhibitors. This is due to the hydrophobicity of its active site. Inhibitors developed were hydrophobic, which aided its permeability, and penetrating the BBB and neuronal membranes was less of a problem. ${ }^{6}$ However, its development was not without challenges. The most serious concern faced was that $\gamma$-secretase had other physiological roles in development 
via Notch signalling regulation. Normally, Notch is cleaved in the Golgi apparatus to produce mature Notch1 protein. Notch-1 undergoes further cleavage to release the Notch intracellular domain (NICD). NICD in turn modifies the transcription of target genes within the nucleus. In PS1 knock-out cells, it was observed that PS1 is vital in the cleavage of APP and Notch. ${ }^{46}$ Hence, the use of $\gamma$-secretases in the treatment of $A D$ can be expected to cause gastrointestinal toxicity, immunosuppression and anaemia due to the role of Notch-1 in haematopoiesis. PS is also involved in several other processes, such as the cellular trafficking of proteins. The knock out of PS would lead to the accumulation of protein fragments within the cell due to interrupted transport between subcellular compartments. It has also been suggested that PS plays a role in homeostasis of $\mathrm{Ca}+{ }^{47}$ Efforts to overcome these challenges come from the development of selective $\gamma$-secretase inhibitors that target cleavage of APP only.

Notch-sparing $\gamma$-secretase inhibitors have also been developed, and these are able to reduce APP proteolysis in the amyloidogenic pathway, thus decreasing $A B$, while preventing the cleavage of Notch. ${ }^{47}$ One of the first compounds with this property was STI571 (Gleevec), an abl kinase inhibitor. STI571 was found to be able to reduce $A ß$ production, albeit weakly, while at the same time did so without causing cleavage of Notch. These agents formed the basis of development of a number of more potent selective inhibitors, and several of such inhibitors have been reported to enter clinical trials. ${ }^{47,48}$

LY-450139 (Semagacestat), a selective $\gamma$-secretase inhibitor that is able to reduce AB production, has been the most widely studied. $L Y-450139$ resulted in dose-dependent decrease in $A B$ concentrations over a period of 6 hours post-administration, with maximum reduction of $40 \%$ relative to the baseline concentration. ${ }^{49}$ This correlated with the fact that semagacestat is absorbed rapidly from the gut, reaching its peak 1.5 hours after administration and the fact that it is mainly excreted renally. In its phase I clinical trial, manageable adverse events were reported. In the Phase II 14-week study performed in 51 mild-to-moderate cases of $A D$, semagacestat was shown to be safe and tolerable. ${ }^{00.51}$ However, during its Phase III trial in 2010, the drug was discontinued due to its detrimental effects on cognitive function of patients receiving it compared to those receiving the placebo. Semagacestat also appeared to be associated with increased risks of skin cancer. Such harmful effects were thought to be due to the involvement of the inhibitor with Notch cleavage and CTFß, a neurotoxic precursor of AB (AlzForum. Drugs in clinical trials. http://www.alzforum.org/drg/drc/detail. asp?id=108. Accessed: 2 Apr 2013). ${ }^{2}$ Setbacks have also been faced in other trials, as seen in Phase III clinical trials of tarenflubril. These trials aimed to determine the efficacy, safety, and tolerability of the drug in the treatment of mild-to-moderate $A D$ patients. However, results failed to show any benefit from tarenglubril in the cognitive function of patients. Possible reasons for this lack of success include insufficient doses and its low penetration across BBB..$^{2}$

BMS-708163 (Avagacestat) is another example of a $\gamma$-secretase inhibitor. Its phase I clinical trial assessed its tolerability, safety and pharmacokinetic properties in young, healthy male volunteers. It reached its peak concentrations rapidly, in about 1.5 hours, with a plasma half-life of about 33 to 34 hours. There was a dose proportional increase in plasma concentration within the range of $0.3-800 \mathrm{mg}$ of the drug. Avagacestat was able to reduce $A ß$ by up to $88 \% 1$ hour post-administration. Some minor side effects included nausea, dizziness and headaches, with no serious side effects or deaths reported. This phase I clinical trial thus demonstrated Avegacestat's tolerability for up to $800 \mathrm{mg}$ dosage, and its suitability for progression into larger clinical studies..$^{33}$ In a phase II clinical trial, 209 patients with mild-to-moderate $A D$ were randomized to receive either the active compound or a placebo. The phase II clinical trial established that daily doses of 25 and $50 \mathrm{mg}$ were indeed weIl-tolerated, whereas 100 and $125 \mathrm{mg}$ doses were not as well tolerated and had a tendency to cause worsening of cognitive function and GI disturbances related to Notch involvement. ${ }^{54}$ These clinical trials have so far established an acceptable dose range for future studies of Avagacestat.

\section{Conclusion}

Taking into account the amount of research that has gone into developing secretase inhibitors, the outcome has not been desirable. The trials so far have come up empty handed, showing no therapeutic improvements in AD patients. As a result, there has been reasonable concern that anti-amyloid therapies may have no therapeutic effect in patients, which would mean decades of wasted research and possibly the targeting of the wrong compounds. Hope that anti-amyloid therapies would have a significant positive impact on AD patients is slowly dissipating. This doubt is further enhanced by the immunization of patients with full length Aß-peptides in an attempt to clear the NPs from the brain. Previously, it was unknown whether active immunization of Aß was able to benefit patients. Thus, a trial was carried out to determine its beneficial effects. In this Phase I, randomized, placebo-controlled follow up on the long term effects of $A B$ immunization (AN1792), data obtained indicated that immunization was indeed able to significantly reduce $A B$ deposition in the $A D$ brain, in comparison to patients receiving the placebo. Disappointingly, despite the pathological improvements observed, the drug failed to produce results of cognitive improvement in these patients. On top of this, some patients even progressed to clinically severe dementia. ${ }^{55}$ What these results imply is that the removal of NPs alone may be insufficient in the disease-modifying treatment of AD. Perhaps challenges against the amyloid hypothesis are reasonable, and other factors such as tau hyperphosphorylation's contribution to $A D$ should be re-examined in greater detail.

However, despite the disappointing lack of improvement in patients with $A B$ generation and deposition, there still is the possibility that these anti-amyloid strategies hold the potential to cure AD. Concluding that anti-amyloid strategies are futile at this point in time would be rash.

First of all, anti-amyloid therapies were indeed successful, as they were able to reduce NPS. Secondly, AB deposition forming NPs was shown to be one of the earliest signs of $A D$, whereas cognitive decline correlated with NFT deposits. ${ }^{28} \mathrm{NPS}$ were thought to be the earliest signs of $A D$, as individuals with trisomy 21 on which the gene for APP is located developed AD much earlier..$^{56}$ Such observations were also made in individuals with familial $A D$. The initial $A B$ deposition is thought to set off 
a series of secondary downstream events, such as the accumulation of tau and inflammatory processes that cause neuronal destruction. ${ }^{57}$ Targeting NPs after a certain point would no longer be beneficial, as other processes become the main propagating factors of the disease. This indicates that perhaps the targeting of NPs much earlier on and in larger doses may be key to anti-amyloid therapies. As such, the way forward in the treatment of $A D$ seems to be the switch from trying to cure $A D$ to instead attempting to prevent it.

\section{References}

1. Povova J, Ambroz P, Bar M, Pavukova V, Sery 0, Tomaskova H, et al. Epidemiological of and risk factors for Alzheimer's disease: A review. Biomed Pap Med Fac Univ Palacky Olomouc Czech Repub. 2012 Jun;156(2):108-14.

2. Yiannopoulou KG, Papageorgiou SG. Current and future treatments for Alzheimer's disease. Ther Adv Neurol Disord. 2013 Jan;6(1):19-33.

3. Förstl H, Kurz A. Clinical features of Alzheimer's disease. EEur Arch Psychiatry Clin Neurosci. 1999;249(6):288-90.

4. Lukiw WJ. Amyloid beta (AB) peptide modulators and other current treatment strategies for Alzheimer's disease (AD). Expert Opin Emerg Drugs. 2012 Mar 23. [Epub ahead of print]

5. Ballard C, Corbett A. Management of neuropsychiatric symptoms in people with dementia. CNS Drugs. 2010 Sep;24(9):729-39.

6. Martâinez A. Emerging drugs and targets for Alzheimer's disease; Volume 1: Beta-Amyloid, Tau protein and glucose metabolism. Cambridge: The Royal Society of Chemistry; 2010.

7. Liberati A, Altman DG, Tetzlaff J, Mulrow C, G $\emptyset$ tzsche PC, loannidis JP, et al. The PRISMA statement for reporting systematic reviews and meta-analyses of studies that evaluate health care interventions: explanation and elaboration. PLoS Med. 2009 Jul 21;6(7):e1000100.

8. Almkvist 0 . Neuropsychological features of early Alzheimer's disease: preclinical and clinical stages. Acta Neurol Scand Suppl. 1996;165:63-71.

9. Galasko D. An integrated approach to the management of Alzheimer's disease: assessing cognition, function and behaviour. Eur J Neurol. 1998 OC$\mathrm{t} ; 5(\mathrm{~S} 4): \mathrm{S} 9-17$.

10. Haupt M, Kurz A. Predictors of nursing home placement in patients with Alzheimer's disease. Int J Geriatr Psychiatry. 1993 Sep;8(9):741-6.

11. Burns A. Psychiatric phenomena in dementia of the Alzheimer type. Int Psychogeriatr. 1992;4 Suppl 1:43-54.

12. Beard CM, Kokmen E, Sigler C, Smith GE, Petterson T, O'Brien PC. Cause of death in Alzheimer's disease. Ann Epidemiol. 1996 May;6(3):195-200.

13. Blennow K, de Leon MJ, Zetterberg H. Alzheimer's disease. Lancet. 2006 Jul 29;368(9533):387-403.

14. Holtzman DM, Bales KR, Tenkova T, Fagan AM, Parsadanian M, Sartorius LJ, et al. Apoliproprotein $\mathrm{E}$ isoform-dependent amyloid deposition and neuritic degeneration in a mouse model of Alzheimer's disease. Proc Natl Acad Sci U S A. 2000 Mar 14;97(6):2892-7.

15. Piaceri I, Nacmias B, Sorbi S. Cenetics of familial and sporadic Alzheimer's disease. Front Biosci (Elite Ed). 2013 Jan 1;5:167-77.

16. Milinois HJ, Florentin MM, Giannopoulos S. Metabolic syndrome and Alzheimer's disease: A link to a vascular hypothesis?. CNS Spectr. 2008 Jul;13(7):606-13.

17. Skoog I, Gustafson D. Update on hypertension and Alzheimer's disease. Neurol Res. 2006 Sep;28(6):605-11.

18. Carlsson CM. Type 2 diabetes mellitus, dyslipidemia, and Alzheimer's disease. J Alzheimers Dis. 2010;20(3):711-22.

19. Naderali EK, Ratcliffe SH, Dale MC. Obesity and Alzheimer's disease: a link between body weight and cognitive function in old age. Am J Alzheimers Dis Other Demen. 2009 Dec-2010 Jan;24(6):445-9.

20. Fratiglioni L, Paillard-Borg S, Winbald B. An active and socially integrated lifestyle in late life might protect against dementia. Lancet Neurol. 2004 Jun;3(6):343-53.

21. Francis PT, Palmer AM, Snape M, Wilcock CK. The cholinergic hypothesis of Alzheimer's disease: a review of progress. J Neurol Neurosurg Psychiatry. 1999 Feb;66(2):137-47.

22. Rogawski MA, Wenk GL. The neuropharmacological basis for the use of Memantine in the treatment of Alzheimer's disease. CNS Drug Rev. 2003 Fall; $9(3): 275-308$.

23. Hsiao K, Chapman P, Nilsen S, Eckman C, Harigaya Y, Younkin S, et al. Correlative memory deficits, $A ß$ elevation, and Amyloid plaques in transgenic mice. Science. 1996 Oct 4;274(5284):99-102. 
24. Lacor PN, Buniel MC, Furlow PW, Clemente AS, Velasco PT, Wood M, et al. Abeta oligomer-induced aberrations in synapse composition, shape, and density provide a molecular basis for loss of connectivity in Alzheimer's disease. J Neurosci. 2007 Jan 24;27(4):796-807.

25. Lambert MP, Barlow AK, Chromy BA, Edwards C, Freed R, Liosatos M, et al. Diffusible, nonfibrillar ligands derived from Abeta1-42 are potent central nervous system neurotoxins. Proc Natl Acad Sci U S A. 1998 May 26;95(11):644853.

26. Mudher A, Lovestone S. Alzheimer's disease-do tauists and baptists finaIly shake hands?. Trends Neurosci. 2002 Jan;25(1):22-6.

27. Trojanowski JQ, Lee VMY. Rous-Whipple Award Lecture. The Alzheimer's brain: finding out what's broken tells us how to fix it. Am J Pathol. 2005 Nov; $167(5): 1183-8$.

28. Tiraboschi P, Hansen LA, Thal LJ, Corey-Bloom J. The importance of neuritic plaques and tangles to the development and evolution of AD. Neurology. 2004 Jun 8;62(11):1984-9.

29. Birks J. Cholinesterase inhibitors for Alzheimer's disease. Cochrane Database Syst Rev. 2006 Jan 25; (1):CDo05593.

30. Courtney C, Farrell D, Gray R, Hills R, Lynch L, Sellwood E, et al. Long-term donepezil treatment in 565 patients with Alzheimer's disease (AD2000): randomized double-blind trial. Lancet. 2004 Jun 26;363(9427):2105-15.

31. Cummings J, Froelich L, Black SE, Bakchine S, Belleli G, Molinuevo JL, et al. Randomized, double-blind, parallel-group, 48-week study for efficacy and safety of a higher-dose rivastigmine patch (15 vs. $\left.10 \mathrm{~cm}^{2}\right)$ in Alzheimer's disease. Dement Geriatr Cogn Disord. 2012;33(5):341-53.

32. Reisberg B, Doody R, Stoffler A, Schmitt F, Ferris S, Mobiue HJ, et al. Memantine in moderate-to-severe Alzheimer's disease. N Engl J Med. 2003 Apr 3;348(14):1333-41.

33. Maidment ID, Fox CG, Boustani M, Rodriguez J, Brown RC, Katona CK. Efficacy of Memantine on behavioural and psychological symptoms related to dementia: a systematic meta-analysis. Ann Pharmacother. 2008 Jan;42(1):328.

34. Zec RF, Burkett NR. Non-pharmacological and pharmacological treatment of the cognitive and behavioural symptoms of Alzheimer disease. NeuroRehabilitation. 2008;23(5):425-38.

35. Vigen CL, Mack WJ, Keefe RS, Sano M, Sultzer DL, Stroup TS, et al. Cognitive effects of atypical antipsychotic medications in patients with Alzheimer's disease: outcomes from CATIE-AD. Am J Psychiatry. 2011 Aug;168(8):831-9.

36. Van Marum RJ. Current and future therapy in Alzheimer's disease. Fundam Clin Pharmacol. 2008 Jun;22(3):265-74.

37. Weksler ME. The immunotherapy of Alzheimer's disease. Immun Ageing. 2004 Nov 12;1(1):2.

38. Chosh AK, Brindisi M, Tang J. Developing ß-secretase inhibitors for treatment of Alzheimer's disease. J Neurochem. 2012 Jan;120 Suppl 1:71-83.

39. Cai H, Wang Y, McCarthy D, Wen H, Borchelt DR, Price DL, et al. BACE1 is the major beta-secretase for generation of Abeta peptides by neurons. Nat Neurosci. 2001 Mar;4(3):233-4.

40. May PC, Dean RA, Lowe SL, Martenyi F, Sheehan SM, Boggs LN, et al. Robust central reduction of amyloid- $B$ in humans with orally available, non-peptidic B-secretase inhibitor. J Neurosci. 2011 Nov 16;31(46):16507-16.

41. Hu X, Hicks CW, He W, Wong P, Macklin WB, Trapp B, et al. Bace1 modulates myelination in the central and peripheral nervous system. Nat Neurosci. 2006 Dec;9(12):1520-5.

\section{Acknowledgments}

I would like to thank Prof T. Francis, PhD, Professor of Neurochemistry, Director of Brains for Dementia Research, KCL, for his guidance in this article.

Conflict of Interest Statement a Funding

The authors have no funding, financial relationships or conflicts of interest to disclose.

\section{Author Contributions}

Conception and design the work/idea: EC. Write the manuscript: EC.

Cite as:

Chou E. Alzheimer's Disease: Current and Future Treatments. A Review. Int J Med Students. 2014 Mar-Jun;2(2):56-63.
42. Velanac V, Unterbarnscheidt T, Hinrichs W, Gummert MN, Fischer TM, Rossner MJ, et al. Bace1 processing of NRG1 type III produces a myelin-inducing signal but is not essential for the stimulation of myelination. Glia. 2012 Feb;60(2):203-17.

43. Zhao G, Liu Z, Ilagan MX, Kopan R. Gamma-secretase composed of PS1/ Pen2Aphra can cleave notch and amyloid precursor protein in the absence of nicastrin. J Neurosci. 2010 Feb 3;30(5):1648-56.

44. Zhang Z, Nadeau P, Song W, Donoviel D, Yuan M, Bernstein A, et al. Presenilins are required for gamma-secretase cleavage of beta-APP and transmembrane cleavage of Notch-1. Nat Cell Biol. 2000 Jul;2(7):463-5.

45. De Strooper B, Saftig P, Craessaerts K, Vanderstichele H, Guhde C, Annaert $W$, et al. Deficiency of presenilin-1 inhibits the normal cleavage of amyloid precursor protein. Nature. 1998 Jan 22;391(6665):387-90.

46. De Strooper B, Annaert W, Cupers P, Saftig P, Craessaerts K, Mumm JS, et al. A presenilin-1-dependent gamma-secretase-like protease mediates release of Notch intracellular domain. Nature. 1999 Apr 8;398(6727):518-22.

47. De Strooper B, Iwatsubo T, Wolfe MS. Presenilins and $\gamma$-secretase: structure, function, and role in Alzheimer disease. Cold Spring Harb Perspect Med. 2012 Jan;2(1):a006304.

48. Netzer WJ, Dou F, Cai D, Veach D, Jean S, Li Y, et al. Gleevec inhibits beta-amyloid production but not Notch cleavage. Proc Natl Acad Sci U S A. 2003 Oct 14;100(21):12444-9.

49. Siemers E, Skinner M, Dean RA, Gonzales C, Satterwhite J, Farlow M, et al. Safety, tolerability, and changes in amyloid beta concentrations after administration of gamma-secretase inhibitor in volunteers. Clin Neuropharmacol. 2005 May-Jun;28(3):126-32.

50. Henley DB, May PC, Dean RA, Siemers ER. Development of semagacestat (LY450139), a functional gamma-secretase inhibitor, for the treatment of Alzheimer's disease. Expert Opin Pharmacother. 2009 Jul;10(10):1657-64.

51. Fleisher AS, Raman R, Siemers ER, Becerra L, Clark CM, Dean RA, et al. Phase 2 safety trial targeting amyloid beta production with a gamma-secretase inhibitor in Alzheimer disease. Arch Neurol. 2008 Aug;65(8):1031-8.

52. Marder K. Tarenflurbil in patients with mild Alzheimer's disease. Curr Neurol Neurosci Rep. 2010 Sep;10(5):336-7.

53. Tong $\mathrm{C}$, Wang JS, Sverdlov 0 , Huang SP, Slemmon R, Croop R, et al. Multicenter, randomized, double-blind, placebo-controlled, single-ascending dose study of the oral $\gamma$-secretase inhibitor BMS-708163 (Avagacestat): tolerability profile, pharmacokinetic parameters, and pharmacodynamic markers. Clin Ther. 2012 Mar;34(3):654-67.

54. Coric V, vanDyck CH, Salloway S, Andreasen N, Brody M, Richter RW, et al. Safety and tolerability of the $\gamma$-secretase inhibitor avagescat in a phase 2 study of mild to moderate Alzheimer disease. Arch Neurol. 2012 Nov;69(11):1430-40.

55. Holmes C, Boche D, Wilkinson D, Yadegarfar G, Hopkins V, Bayer A, et al. Long-term effects of Abeta42 immunisation in Alzheimer's disease: follow-up of a randomised, placebo-controlled phase I trial. Lancet. 2008 Jul 19;372(9634):216-23.

56. Lippa CF, Nee LE, Mori H, St George-Hyslop P. Abeta-42 deposition precedes other changes in PS-1 Alzheimer's disease. Lancet. 1998 0ct 3;352(9134):11178.

57. St George-Hyslop PH, Morris JC. Will anti-amyloid therapies work for Alzheimer's disease?. Lancet. 2008 Jul 19;372(9634):180-2. 